\title{
El olivo monumental plantado por San Martín de Porres
}

\section{The monumental olive tree planted by Saint Martín de Porres}

\author{
Jorge Gianfranco Vargas Flores* \\ Escuela Profesional de Turismo y Hotelería, \\ Universidad de San Martín de Porres, Perú
}

\section{Resumen}

El estudio aborda la constatación científica del olivo plantado por San Martín de Porres. Se indagó, en las fuentes históricas, los documentos del proceso de beatificación de fray Martín de Porres, donde se narra que, efectivamente, Martín de Porres plantó algunos olivos en 1637 en una zona que hoy forman parte del bosque El Olivar de San Isidro. A través del «Modelo Santander» se determinó la edad de un árbol de olivas que fuera plantado en aquel entonces por San Martín de Porres, en el tradicional bosque El Olivar de San Isidro de la ciudad de Lima. Mediante un acuerdo para el estudio de este olivo monumental, entre la Universidad de San Martín de Porres y la Universidad Politécnica de Madrid, se determinó el número de años que tiene este olivo.

Palabras clave: olivo monumental, «Modelo Santander», San Martín de Porres. 


\begin{abstract}
Abstact
The study deals with the scientific verification of the olive tree planted by Saint Martín de Porres. Historical source documents on Brother Martín de Porres's process of beatification evidence that, indeed, he planted some olive trees in 1637 in an area that today is part of the traditional El Olivar de San Isidro grove in the city of Lima. Using the Santander model, the age of an olive tree planted in those days by Saint Martín de Porres in said grove was determined, thanks to an agreement between the Universidad San Martín de Porres and the Universidad Politécnica de Madrid to study this monumental olive tree.
\end{abstract}

Keywords: monumental olive tree, Santander model, Saint Martín de Porres.

Mediante la investigación, titulada El cultivo del olivo en el Perú: Patrimonio olivarero sudamericano, que viene desarrollando el investigador y experto oleícola Gianfranco Vargas Flores, desde el Instituto de Investigación de la Escuela de Turismo y Hotelería de la Universidad de San Martín de Porres; se ha logrado identificar e iniciar la puesta en valor de la cultura olivarera peruana y los olivos singulares de América del Sur.

Uno de los hitos trascendentales de este proyecto es la constatación científica del olivo plantado por San Martín de Porres. A través del estudio historiográfico mediante fuentes primigenias sobre el proceso de beatificación de San Martín de Porres, y aplicando el «Modelo Santander» para la datación de olivos, tecnología de punta desarrollada por ingenieros de la Universidad Politécnica de Madrid, se pudo determinar la edad y la ubicación exacta del único árbol de olivo sobreviviente que fuera plantado por santo peruano en el actual bosque El Olivar de San Isidro (Soria, Prieto, \& Saiz 2016). 
En cuanto a las fuentes históricas, nos remitimos a los documentos de la beatificación del actual santo, en los que el asistente del fraile, Juan Vázquez Parra, brinda testimonio sobre un hecho milagroso:

En el mes de agosto de 1637, Martín y Juancho se trasladaron a la hacienda de Limatambo (hoy El Olivar de San Isidro), donde plantaron 700 esquejes de olivo en menos de 15 días. Sucedió que las ramas retoñaron hojas al tercer día de riego, es por esta acción sobrenatural que fue considerada un milagro durante el proceso de beatificación de Martín de Porres Velázquez. (Secretariado «Martín de Porres», 1960)

A continuación, se detalla la recreación de la plantación de olivos realizada por San Martín de Porres, extraído del Proceso de Beatificación de Fray Martín de Porres, Volumen I. Proceso Diocesano Años 1660, 1664, 1671 (Secretariado «Martín de Porres», 1960). Según el citado testimonio, una mañana de invierno limeño, durante el mes de agosto de 1637, San Martín y Juan Vázquez Parra (Juancho como llamaba fray Martín a su asistente) salieron del Convento del Santísimo Rosario, llevando en sus alforjas una buena carga de semitas o llamado también pan basto.

Al salir por la puerta falsa del citado convento, el joven Juancho, llevado por su natural curiosidad, le preguntó a fray Martín:

— Juancho: «iA dónde vamos, Padre?».

- Fray Martín: «...a Limatambo, que voy hacer un servicio a Dios, hay muchos novicios y tenemos que hacer bastante allá, más de dos meses».

— Juancho: «Padre, ¿qué hemos de hacer que tanto hemos de tardar?».

- Fray Martín: «Es que poda fray Francisco el viejo olivar y cortaremos estacas, para desde el camino real hasta el molino hacer un nuevo olivar, para que estos muchachos tengan en el tiempo de adelante con qué poder pasar, que el olivar que hoy hay es ya viejo y se irá criando otro nuevo y de aquí a treinta años, que ya éstos serán hombres maduros, dirán que Dios perdone a quien plantó este olivar». 
Las semitas que sacaron del convento las repartió el venerable fray Martín a los muchachos que encontraba por el camino, diciéndoles:

— Fray Martín: «Algún día me ayudaréis a trabajar».

El plantío de olivos en Limatambo (actual olivar de San Isidro).

Llegaron a Limatambo y entraron en el viejo olivar. Fray Martín pidió al P. fray Francisco, que era el que podaba las plantas, que le fuese apartando varas, porque tenía dispuesto el plantar un olivar desde el camino real hasta el molino.

Riéndose fray Francisco, le responde a fray Martín:

— Fray Francisco: «ia dónde podía haber tantas varas?»

— Fray Martín: «No se aflija, que la Providencia de Dios es grande, que con los muchachos que había en casa había harto para que le hiciesen agujeros; que su paternidad, mandase que con cuatro muías y dos negros se las fuesen llevando allí».

Salió el P. fray Martín a comenzar los hoyos con tres barretinas y el primer día se hicieron noventa hoyos, poco menos de media vara de hondo y una cuarta de ancho, y a este paso fueron trabajando toda la semana.

Juan Vázquez testimonia que el sábado, por la mañana, fray Martín se levantó dando gracias a Dios (como se citó en Secretariado «Martín de Porres», 1960):

Porque le había dejado llegar al día que comenzaba a plantar su olivar y comenzando a poner varas, fuimos plantando todo aquel día; y al día siguiente del lunes, del medio día para arriba, comenzó a dar agua a todas las varas que tenía plantadas y fue providencia de Dios, que, al tercer día de regadas, había una cuarta de retoño en cada una. Fuimos prosiguiendo con la planta. A los quince días había plantado setecientas plantas, lo cual en un mes acabó de hacer su olivar, que hoy es prodigio el verlo. (p. 398) 


\section{El método de datación aplicado: «Modelo Santander»}

El olivo plantado por San Martín de Porres, se ubica en el perímetro comprendido entre la avenida Los Incas y las calles Choquehuanca, Ántero Aspíllaga y Paillardelle. Nunca antes, se había realizado un estudio científico para determinar la antigüedad de los olivos plantados en el Olivar de San Isidro, ni muchos menos, para identificar a aquellos cuya antigüedad coincide con la época en que San Martín de Porres los plantó en ese lugar.

Con el fin de determinar la edad de los olivos sin causarles ningún tipo de daño, se optó por hacer uso del «Modelo Santander» para la datación de olivos o árboles monumentales. Un procedimiento que se viene utilizando en el Mediterráneo, como alternativa a los métodos clásicos, como el carbono 14, radiocarbono o la medición de los anillos de crecimiento con barreno, desmiembran madera del tronco base, haciendo un daño irreparable al árbol en estudio, sobre todo al momento de extraer el muestreo de los radios internos del tronco.

$\mathrm{El}$ «Modelo Santander» fue desarrollado por los catedráticos e ingenieros en dasometría (mediciones vegetales) Soria et al. (2016), docentes de la Escuela Técnica Superior de Ingenieros de Montes de la Universidad Politécnica de Madrid. Mediante su trabajo, se ha podido determinar que el olivo estudiado fue plantado por San Martín de Porres. Según certificación emitida por el laboratorio de dasometría de la Universidad Politécnica de Madrid, utilizando como referencia el «Modelo Santander» de datación desarrollado por dicha universidad.

Aplicando la citada metodología, la cual se basa en el cálculo de las coordenadas de los puntos del perímetro externo, se procede a ajustar una cinta métrica al contorno del tronco, obteniendo así un perímetro exacto del mismo, que incluye todas las irregularidades que caracteriza a los olivos.

El perímetro del olivo (medido a la altura del tronco en que es más delgado y si no presenta esa delgadez a 1.30 metros sobre el nivel del suelo) con la cinta métrica lo más pegada al tronco y una foto del olivo con una mira de 1 metro de longitud, perpendicular y pegada al tronco, por el lado que tenga mayor dimensión el olivo. Se debe tener la seguridad de que se 
trata de un solo tronco, ya que muchas veces son olivos de grandes dimensiones de troncos unidos, por lo que su edad no es la correspondiente a su tamaño, sino la del tronco más viejo.

A continuación, se colocan unas miras graduadas (con una cinta de cuatro lados iguales se demarca el contorno del olivo, formando de esta manera un cuadrado alrededor del mismo) formando un cuadrado alrededor del ejemplar. Seguidamente se realizan, con distanciómetro láser o telemetría láser, las mediciones correspondientes al perímetro exterior con todas sus irregularidades, obteniéndose la distancia desde el punto a medir hasta la mira.

Se repite este procedimiento en los cuatro lados del cuadrado, de manera que de cada uno de los puntos del perímetro se obtendrán al menos dos coordenadas. De esta manera, se puede obtener la sección completa del árbol mediante la sucesión continua de los puntos.

Una vez obtenida la sección, se calcula su área, así como el centro de gravedad, con lo que se puede calcular el radio máximo, mínimo, medio (semisuma del radio máximo y mínimo) y equivalente de la sección considerada como circular; y, a partir de toda esta información incluyendo los patrones de crecimiento (además de la climatología, edafología de la zona y espaciamiento de los olivos), conocer la edad del árbol.

Los autores Soria et al. (2016) certificaron oficialmente las edades aproximadas de los olivos monumentales. Detallando que, de acuerdo a las mediciones y estudios realizados en el olivo monumental, localizado en el Bosque del Olivar del distrito de San Isidro, de la ciudad de Lima (Perú), este cuenta con aproximadamente 374 anos de edad.

Evaluando la edad resultante (374 años), obtenida mediante el «Modelo Santander», los autores Soria et al. (2016) certificaron oficialmente que el olivo identificado como monumental en el Olivar de San Isidro, fue plantado por San Martín de Porres, mencionando que el laboratorio de dasometria de la Universidad Politécnica de Madrid, detalla que el olivo es de 1637 y tiene 380 años; la diferencia está en el factor o índice de Paterson, si ha regado un poco mas o un poco menos (Soria et al., 2016). Es decir, el cálculo 
del índice de Paterson o indice C. V. P. (clima - vegetación - producción) permitió estimar el nivel máximo de la masa de madera del árbol, y su crecimiento fue ha acondicionado a la cantidad de riego recibido (Paterson, 1956).

Es decir, en cuanto a la diferencia del resultado obtenido (374 años) con la sustentación histórica (380 años), Soria et al. (2016) mencionan que todo es consecuencia por el riego inadecuado del olivo durante las últimas décadas, creciendo de manera irregular, sin conocer la cantidad exacta litros/ años de riego. A pesar de ello, el método aplicado no se aleja del ponderado cronológico real, son solo seis años de diferencia del tiempo atestiguado por Vásquez de Parra.

Finalizando el estudio, Soria et al. (2016) detallan que el titular (Antonio Prieto) de la Escuela Técnica Superior de Ingenieros de Montes, Forestal y del Medio Natural (ETSIMFMN) de la Universidad Politécnica de Madrid constata la coincidencia de la edad de 374 años obtenida mediante la aplicación del «Modelo Santander», indicando que el citado olivo monumental fue plantado por San Martín de Porres en 1637, y se encuentra dentro del intervalo de origen posible (380 años).

\section{Referencias}

Paterson, S. S. (1956). The forest area of the world and its potential productivity. Göteborg: Royal University of Göteborg.

Secretariado «Martín de Porres». (1960). Proceso de beatificación de fray Martín de Porres (Vol. I). Proceso diocesano años 1660, 1664, 1671. Recuperado de http://traditio-op.org/santos/San\%20Martin\%20de\%20Porres/ Proceso $\% 20$ de $\% 20$ Beatificacion $\% 20$ de $\% 20$ fray $\% 20$ Martin $\% 20$ de $\% 20$ Porres $\% 200$ P.pdf

Soria, S., Prieto, A., \& Saiz, J. (2016). El «Modelo Santander» de determinación de la edad de olivos monumentales. Mercacei magazine, 87, 114-121. 